\title{
Grain kinematics during stress relaxation in sand: not a problem for X-ray imaging
}

\author{
Jelke Dijkstra ${ }^{1, *}$, Edward Andò ${ }^{2}$, and Christophe Dano $^{2}$ \\ ${ }^{1}$ Chalmers University of Technology, Arch. \& Civil Eng., SE-41296, Gothenburg, Sweden \\ ${ }^{2}$ Univ. Grenoble Alpes, CNRS, Grenoble INP 3SR, F-38000 Grenoble, France
}

\begin{abstract}
X-ray tomography is a very valuable tool for studying the full-field 3D deformation of granular materials. The requirement to stop loading and scan a given state (assumed to be stationary) used in most approaches implies unavoidable stress relaxation during scanning. Since scanning times on laboratory tomographs are normally in the order of 1 hour, the strength of the assumption of a stationary state cannot be tested, which introduces some potential weakness in the interpretation of the rich micro-mechanics observed. This paper presents the kinematics of relaxation of a dry natural sand in a typical oedometric cell used for X-ray scanning, using a synchrotron X-ray source to provide scanning times of around 3 minutes, at two different magnifications. This allows the relaxation of the cell \& sand system for the first time to be quantified. Advanced image correlation tools are used to quantify the rearrangements of the soil skeleton during loading and the subsequent relaxation. The results indicate that the magnitude of grain displacements during relaxation, associated to $\approx 4 \%$ reduction in externally measured axial stress under oedometric loading, falls below $0.01 \mathrm{D}_{50}$. It can, therefore, be concluded that the relaxation step required prior to an X-ray scan during an in-situ geomechanical experiment on dry sand does not lead to appreciable uncertainties.
\end{abstract}

\section{Introduction}

Micro-mechanical approaches to the description and modelling of natural granular soils are fuelled experimentally by the relative ease-of-access of laboratory X-ray tomography $[1,2]$. This valuable tool allows a $3 \mathrm{D}$ field of $\mathrm{X}$ ray attenuation to be measured repeatedly during loading, allowing deformation processes to be monitored in space and time. The main challenge in laboratory Xray tomography is to balance the spatial and the temporal scales of observation with the processes in the experiment (and with acceptable noise in the measurement). The scanning time of most laboratory based X-ray tomographs over quite a wide range of sample sizes is around 1 hour for a level of noise acceptable for most types of quantification. However, the majority of processes in natural granular soils falls within this 1 hour scanning time. Consequently, most geomechanical tests on granular soils in an X-ray tomograph need to be adapted to facilitate the scans. For displacement controlled mechanical loading, such as 1D compression tests, uniaxial loading and triaxial loading paths, this often is accommodated by momentarily pausing the test during the scan. In this context pausing the test means: (i) stopping the loading ram, (ii) allowing the stress in the sample to somewhat relax as judged from monitoring the load cell connected to the ram (iii) perform an X-ray scan, and finally (iv) continue the displacement controlled loading sequence.

*e-mail: jelke.dijkstra@chalmers.se
The main assumption is that during the scan following the stress relaxation a stationary state is reached, i.e., the grains do not move (or change shape) appreciably, leading to a reliable reconstruction of the 3D field of X-ray attenuation. Furthermore, it is implicitly assumed that the state of the sample, as captured during the scan closely resembles the state of the sample at the particular stage during the test before pausing, stress relaxation and scanning. These two assumptions are pragmatic at a time when the novelty of the observed micro-mechanical mechanisms trumps the attainable accuracy. Stop-and-scan X-ray scanning has become a main-stream quantitative tool used to study increasingly smaller features and more subtle mechanisms, and therefore further scrutiny of the assumptions made is required.

This paper presents a study of the grain kinematics during stress relaxation under oedometric conditions by exploiting the high-speed and high-resolutions possible at the X-ray microtomography beamline ID19 at the European Synchrotron Facility.

\section{Experimental methods}

\subsection{Material}

Hostun HN31, which is an uniform angular coarse grained material that is often used for in-situ X-ray experiments, has been used for the reported test. The average grain size of $D_{50}=0.328 \mathrm{~mm}$ makes it well suited for laboratory based X-ray experiments and hence poses no issues 
for high resolution tomography at the synchrotron beamline. A dry sample, with density $\rho=1650 \pm 90 \mathrm{~kg} \mathrm{~m}^{-3}$, diameter $D=15 \mathrm{~mm}$, and height $h=6.1 \mathrm{~mm}$ is prepared in the PEEK confining cell by combining air pluviation with a zero drop height using a funnel, and subsequent compaction in the cell. The sample diameter and grain size are chosen such that wall-friction effects are minimised, the sample height $h_{\text {sample }}$ is $>5 D_{\max }$. Furthermore, the density chosen represents a state that will be most prone to relaxation.

\subsection{Loading system}

Fig. 1 shows the test setup used. The 1D oedometer cell and loading cap are machined from Polyether Ether Ketone (PEEK) to ensure sufficient stiffness and low X-ray attenuation, as well as that the cylindrical shape makes the sample most suitable for X-ray tomography. The cell used is the same cell used in [3] and has a minimum wall (base) thickness of $15 \mathrm{~mm}$. A mechanical actuator capable of applying $15 \mathrm{kN}$ compressive force, with a minimum loading rate of $2 \mu \mathrm{m} / \mathrm{min}$, is used to apply the axial loads. As indicated in Fig. 1 the actuator is mounted on a cylindrical reaction frame made out of aluminium, inside which the scan takes place. The mechanical design of the actuator, the stepper motor + mechanical gearbox, ensures minimal backlash when pausing the actuator during a scan, i.e. no additional control loop is required to keep the axial displacement applied on the loading ram constant.

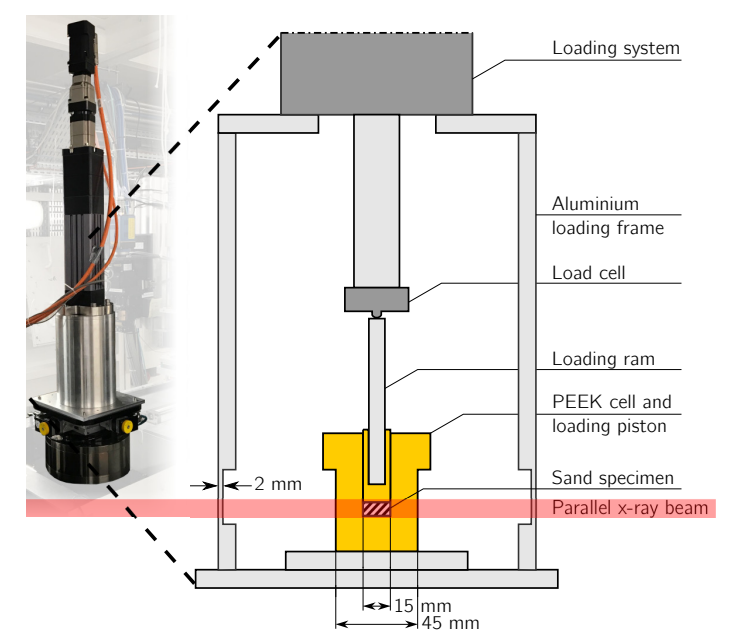

Fig. 1. Test setup for 1D oedometric loading; The Peek cell and loading ram are surrounded by an aluminium support frame for the actuator stack.

\subsection{X-ray tomography}

At the id19 tomography beamline at the ESRF, high speed and high resolution tomography is nowadays routine. In this experimental work, two different tomography measurement setups are used on the same sample: in the first configuration the "full-sample" is scanned with a pixel size of $6.5 \mu \mathrm{m} / \mathrm{px}$ where the entire sample is in the field of view (deep within the loading frame). Second, during the highresolution scans with a pixel size of $0.65 \mu \mathrm{m} / \mathrm{px}$ and a field of view of within, an area of $1.3 \mathrm{~mm} \times 1.3 \mathrm{~mm} \times 1.3 \mathrm{~mm}$ centred on the axis of the specimen is measured. Thanks to the high photon flux available at id19 [4] in both configurations, the scans take fewer than five minutes. Since the beam is parallel, radiographies are only collected over $180^{\circ}$. 3D reconstructions of the scans are made with the "Paganin" phase-contrast retrieval algorithm [5], which significantly improves contrast at the expense of a little blur.

\subsection{Full-field analysis}

The full-specimen X-ray tomography volumes are amply of sufficient resolution to easily identify individual particles. In fact, in this work spatial resolution is sacrificed in favour of noise reduction by averaging $2 \times 2 \times 2$ pixels together into a new pixel, thus doubling the pixel size to $13 \mu \mathrm{m} / \mathrm{px}$. The solid phase in the tomography volumes is identified by applying the threshold given by the classical Ostu method. Thereafter, the sPAm package [6] which itself relies on the ITK morphological watershed [7, 8] is used to identify and uniquely number (segmentation) individual particles in a full-specimen volume taken as the reference.

Thereafter, Discrete Digital Image Correlation (as implemented in the SPAM package, inspired by [9]), is used to measure particle displacements between the segmented reference state and a different state, as captured in another full-specimen scan.

The small rearrangements that are expected during stress relaxation require the error of the displacement measurement to be studied with care. To this end, an specimen of epoxied Hostun sand grains - in order to guarantee no relative displacements of the grains - was prepared and scanned in two different positions differing by a small translation (applied to the entire system) in the vertical $z$ direction. The displacement of grains is measured and is considered to be the net of segmentation errors, interpolation, as well as noise and artefacts in the reconstruction of the X-ray attenuation field.

The measured $x, y, z$ displacements of 8194 grains minus the corresponding median value (simply to allow easier comparative plotting for the $z$-displacement) in each direction is shown as a histogram (bin width $=0.001 \mathrm{px}$ ) in Figure 2 left. The standard deviation resulting from the Gaussian fits of each component presented is less than $0.02 \mathrm{px}$ in each direction. This is therefore an eminently sub-pixel measurement whose precision will allow some fine details of granular kinematics to be revealed.

\section{Relaxation test}

An oedometric test is performed in-situ on the tomography beamline. The sample is compressed to $625 \mathrm{kPa}$ axial stress (progressively slowing loading rate in order not to overshoot the desired stress level), after which the displacement of the ram is stopped, and an initial full-sample scan (44-LR) on Figure 3 (right) is immediately started. 

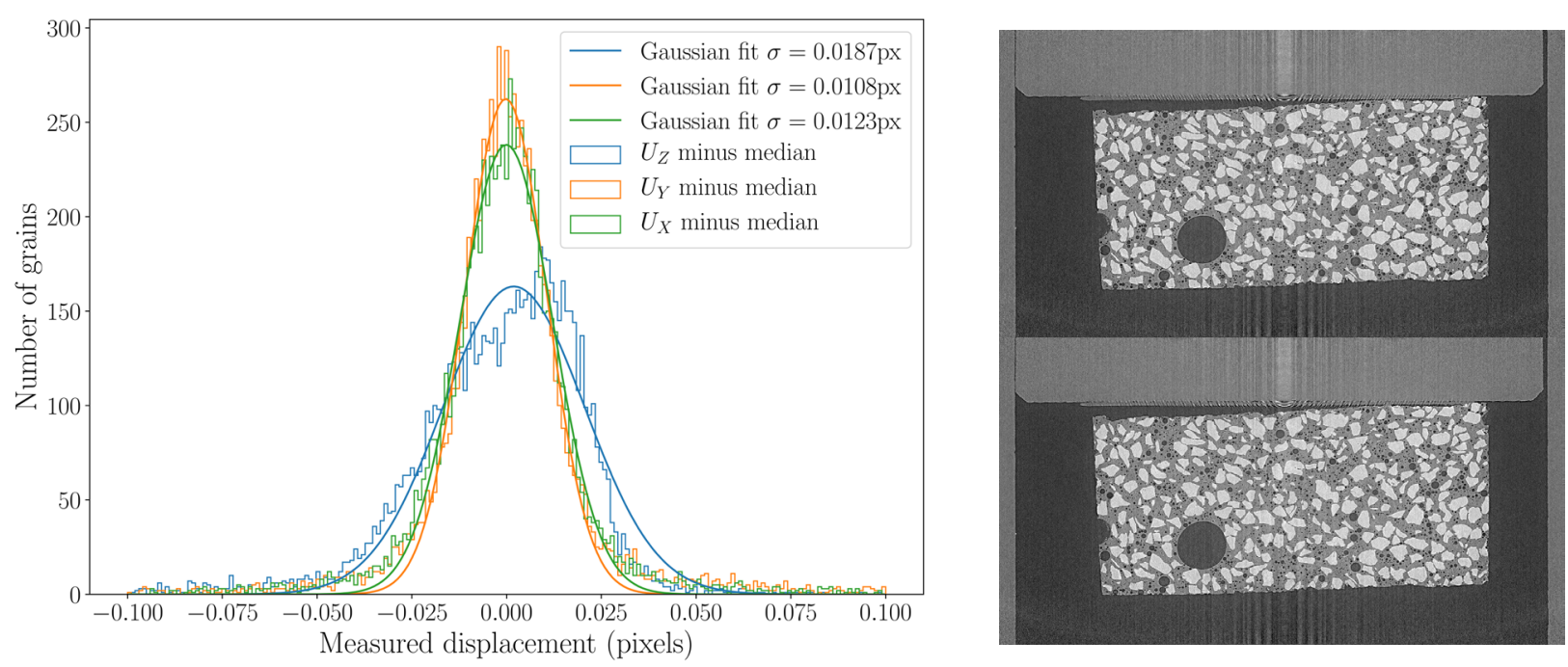

Fig. 2. Histogram and Gaussian fit of displacement errors from DVC analysis of two subsequent LR tomographies (left) of a translated epoxy sample with bonded sand grains (right).
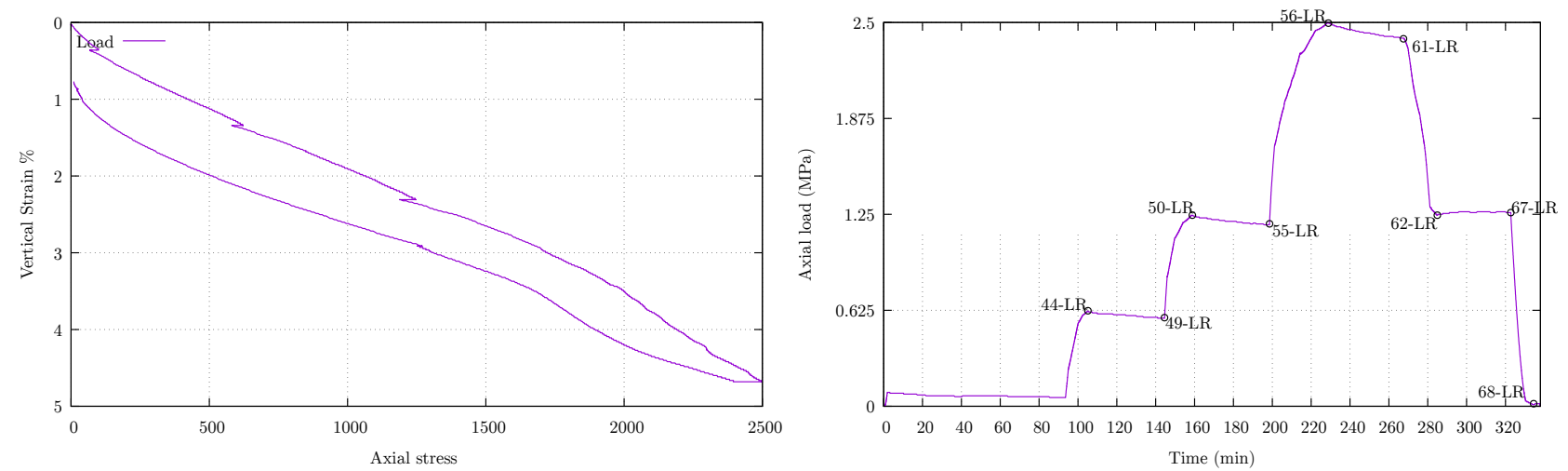

Fig. 3. Axial load - vertical strain curve (left) and time-axial load curve (right) measured on the top cap of the sample. The additional labels annotated in the time-axial load curve correspond to the X-ray scans performed.

A series of high-resolution scans are thereafter acquired for around 40 minutes, after which another full-sample scan is performed. Thereafter the load is doubled to $1250 \mathrm{kPa}$ and the same scanning strategy is repeated, starting with scan 50-LR and finishing with 55-LR. Thereafter, the load is doubled again to $2500 \mathrm{kPa}$ and the same scanning strategy is repeated, starting with scan 56-LR and finishing with 61-LR. Using scan 44-LR as a reference configuration, grains are tracked into all other full-specimen scans. The externally measured axial stress reduces $4.3 \%$, $4.5 \% \& 4.4 \%$ for each subsequent relaxation stage, respectively.

Figure 4 presents displacement vectors over the three different stress-relaxation periods in a thin central slice, of about 6 grains thick, in the middle of the specimen, vector lengths are exaggerated 100 times for visibility.

Over the first relaxation step at $625 \mathrm{kPa}$, a displacement field is measured, revealing that some small grain displacements belie the loss of stress in the granular system. The maximum (inward) grain displacement measured in this increment is in the order of $0.3 \mathrm{px}(4 \mu \mathrm{m}$, or $\left.0.01 \mathrm{D}_{50}\right)$. In this step with zero imposed external displacement from the actuator, there appears to be, at the particle level, a continued settlement of the top cap, as well as some elastic rebound of the bottom of the cell. Furthermore, in addition to the bottom, the walls of the cell also appear to move inward, hence significantly pushing the grains together. During this relaxation step the elastic rebound of the cell walls appear to densify the sample.

The displacement fields measured during the following relaxation steps $-50 \rightarrow 55$ and $56 \rightarrow 61$ are computed by subtracting the displacements measured from 44 to the beginning of the step, from the displacements measured from 44 to the end of the step (thus doubling the expected error in the displacement measurement). During the relaxation step at $1250 \mathrm{kPa}$ practically no grain kinematics above the noise level of the measurement are detected, whilst at $2500 \mathrm{kPa}$ some axial displacements that still are significantly smaller than in the relaxation step at $625 \mathrm{kPa}$ are detected. 


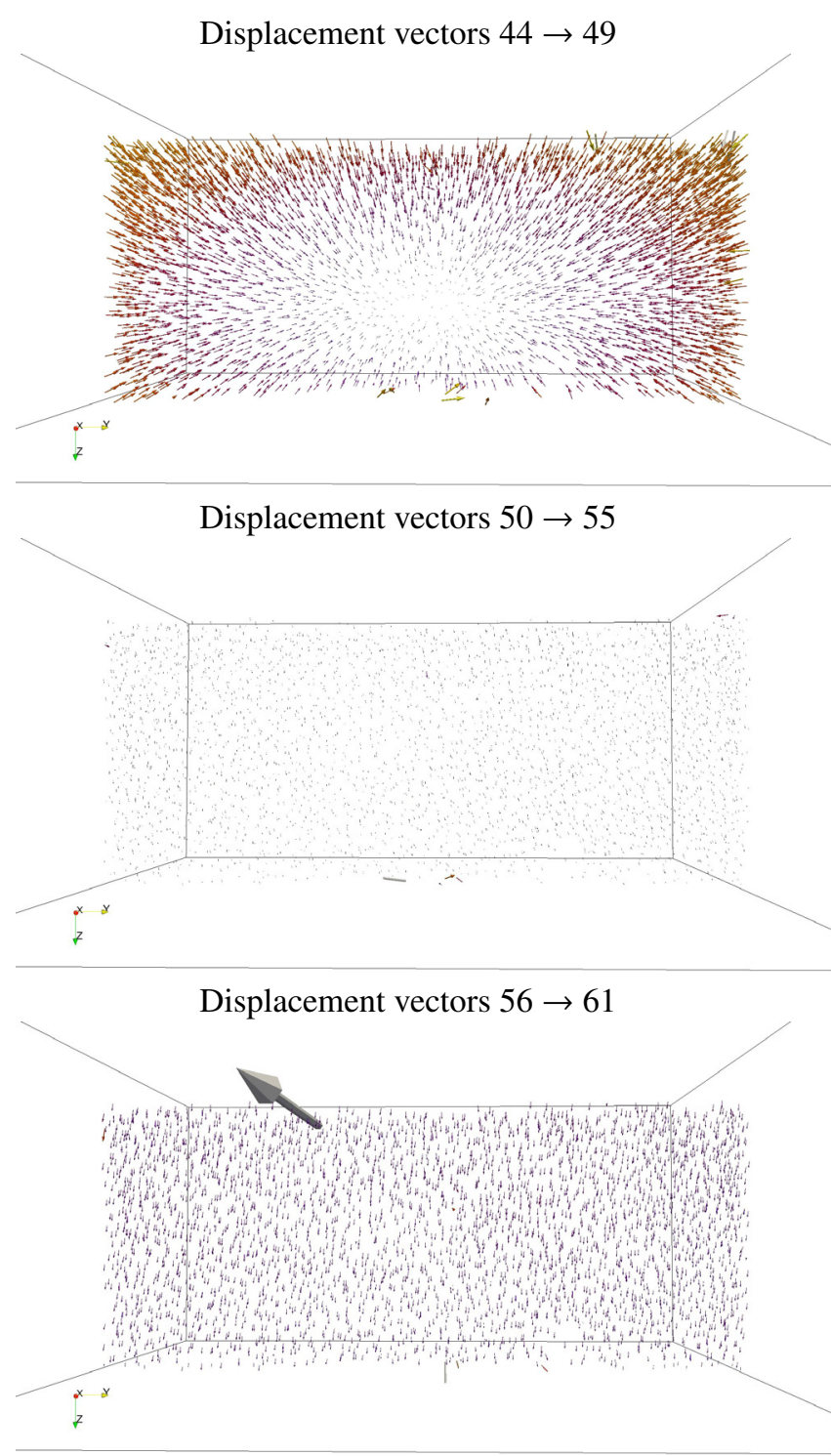

Fig. 4. Displacement vector plots for a slice in the middle of the volume of about 6 grains thick, colours corresponding to the norm of the vector. Vector lengths are scaled up 100 times for visibility.

\section{Discussion \& Conclusions}

In the first stage of loading, it appears that the unloading stiffness of the granular material (estimated -using a $v=$ 0.3 - at $E^{\prime} \approx 57 \mathrm{MPa}$ ) is significantly lower than that of the PEEK confining cell $(E \approx 4 \mathrm{GPa})$. In the two subsequent relaxation steps, at a higher confining stress, the relative reduction in axial stress is similar, but results in significantly smaller grain displacements. It is postulated that in those two relaxation steps the stiffness of the grain assembly is approaching the same order of magnitude as that of the PEEK cell, and that most unloading occurs at the particle-particle contacts. The compressive loading path reduces the void ratio. Consequently, the results for each relaxation step are not solely stress-dependent.

These unique test results show that relaxation effects on the grain assembly scale are negligible, i.e $0.01 \mathrm{D}_{50}$ for the largest detected displacements. Hence, geomechani- cal experiments using laboratory based X-ray tomography that needs a mechanical relaxation step prior to an X-ray scan, provides reliable information reflecting the state in the sample before relaxation. Furthermore, a stationary state is maintained during the X-ray scan. These findings reinforce the use of laboratory based X-ray tomography as a very valuable tool for studying the full-field 3D deformation of granular materials.

\section{References}

[1] E. Andò, S.A. Hall, G. Viggiani, J. Desrues, P. Bésuelle, Acta Geotechnica 7, 1 (2012)

[2] V. Cnudde, M.N. Boone, Earth-Science Reviews 123, 1 (2013)

[3] G. Guida, F. Casini, G. Viggiani, E. Andò, G. Viggiani, Géotechnique Letters 8, 155 (2018)

[4] T. Weitkamp, P. Tafforeau, E. Boller, P. Cloetens, J.P. Valade, P. Bernard, F. Peyrin, W. Ludwig, L. Helfen, J. Baruchel, Status and evolution of the ESRF beamline ID19, in AIP Conference Proceedings (AIP, 2010), Vol. 1221, pp. 33-38

[5] S. Mayo, T. Davis, T. Gureyev, P. Miller, D. Paganin, A. Pogany, A. Stevenson, S. Wilkins, Opt. Express 11, 2289 (2003)

[6] E. Andø, R. Cailletaud, E. Roubin, O. Stamati, the spam contributors, spam: The software for the practical analysis of materials, https://ttk. gricad-pages.univ-grenoble-alpes.fr/spam/ (2017-)

[7] L.I.W. Schroeder, L. Ng, J. Cates et al., The Insight Consortium (2003)

[8] R. Beare, G. Lehmann, Insight J. 92, 1 (2006)

[9] S. Hall, N. Lenoir, Y. Pannier, J. Desrues, M. Bornert, G. Viggiani, P. Bésuelle, M. Di Michiel, J. Otani, Characterisation of localised deformation in granular geomaterials using X-ray (micro) tomography and $3 D$-volumetric digital image correlation (Photomechanics 2008, 2008) 